\title{
$L^{1}$-SPECTRUM OF BANACH SPACE VALUED ORNSTEIN-UHLENBECK OPERATORS
}

\author{
ROSTYSLAV V. KOZHAN
}

\begin{abstract}
We characterize the $L^{1}\left(E, \mu_{\infty}\right)$-spectrum of the Ornstein-Uhlenbeck operator $L f(x)=\frac{1}{2} \operatorname{Tr} Q D^{2} f(x)+\langle A x, D f(x)\rangle$, where $\mu_{\infty}$ is the invariant measure for the Ornstein-Uhlenbeck semigroup generated by $L$. The main result covers the general case of an infinite-dimensional Banach space $E$ under the assumption that the point spectrum of $A^{*}$ is nonempty and extends several recent related results.
\end{abstract}

\section{INTRODUCTION AND RESULTS}

In this paper we investigate spectral properties of the generator of the transition semigroup associated with the stochastic linear Cauchy problem

$$
\left\{\begin{aligned}
d U_{t} & =A U_{t} d t+B d W_{t}^{H} \\
U_{0} & =x
\end{aligned}\right.
$$

where $A$ is the generator of a $C_{0}$-semigroup $(S(t))_{t>0}$ on a real Banach space $E, B$ is a nonzero bounded operator from a real Hilbert space $H$ into $E,\left(W_{t}^{H}\right)_{t \geq 0}$ is an $H$-cylindrical Wiener process, and $x \in E$. The problem is a natural infinite-dimensional generalization of the Langevin equation and arises in many applications, for example in optimal control theory and interest rate models, see [5, 6].

It is well known [1, 6] that the problem (1.1) admits a unique weak solution $\mathbf{U}=$ $\left(U_{t}(x)\right)_{t \geq 0}$ if and only if for all $t \in(0, \infty)$ there exists a centered Gaussian Radon measure $\mu_{t}$ on $\mathrm{E}$ with covariance operator $Q_{t} \in \mathcal{L}\left(E^{*}, E\right)$ given by

$$
\left\langle Q_{t} x^{*}, y^{*}\right\rangle=\int_{0}^{t}\left\langle S(s) B B^{*} S^{*}(s) x^{*}, y^{*}\right\rangle d s, \quad x^{*}, y^{*} \in E^{*},
$$

and in this case the solution can be represented in the form

$$
U_{t}(x)=S(t) x+\int_{0}^{t} S(t-s) B d W_{t}^{H} .
$$

The process $\mathbf{U}$ in (1.3) is Gaussian and Markov and its transition semigroup $\mathbf{P}=(P(t))_{t \geq 0}$ (the Ornstein-Uhlenbeck semigroup) is defined on bounded Borel functions on $E$ by

$$
(P(t) f)(x):=\mathbb{E}\left(f\left(U_{t}(x)\right)\right)=\int_{E} f(S(t) x+y) d \mu_{t}(y) .
$$

2000 Mathematics Subject Classification. 47A10, 47D06, 60H15. 
Assume that the limit $Q_{\infty}:=\lim _{t \rightarrow \infty} Q_{t}$ exists in the weak operator topology of $\mathcal{L}\left(E^{*}, E\right)$ and that there exists a centered Gaussian Radon measure $\mu_{\infty}$ with covariance operator $Q_{\infty}$. Under this assumption the measure $\mu_{\infty}$ is invariant for $\mathbf{P}$ (see [1, 6]), i.e.

$$
\int_{E} P(t) f(x) d \mu_{\infty}(x)=\int_{E} f(x) d \mu_{\infty}(x), \quad t \geq 0 .
$$

Throughout the paper we assume $\mu_{\infty}$ to be nondegenerate.

The above equality easily implies (see [13, Thm XIII.1]) that the semigroup $\mathbf{P}$ has a unique extension to a strongly continuous contraction semigroup on $L^{p}\left(E, \mu_{\infty}\right), p \in$ $[1, \infty)$, which we also denote by $\mathbf{P}$. Properties of $\mathbf{P}$ in $L^{p}\left(E, \mu_{\infty}\right)$ for $p \in(1, \infty)$ have been extensively investigated in the literature (see [3, 4, 5, 9, 10] and references therein). Properties of $\mathbf{P}$ in $L^{1}\left(E, \mu_{\infty}\right)$ turn out to be completely different from those in the spaces $L^{p}\left(E, \mu_{\infty}\right), p \in(1, \infty)$. In particular for $p=1$ the semigroup $\mathbf{P}$ loses its regularity properties, which it possesses in the case $p \in(1, \infty)$ (see [4, 5, 8]), and the spectrum of its generator, which is $p$-independent for $p \in(1, \infty)$ (see [9, 10]), changes drastically.

The key issue investigated in the present paper is the structure of the spectrum of the generator $L$, called the Ornstein-Uhlenbeck operator, of this semigroup in $L^{1}\left(E, \mu_{\infty}\right)$. Denote by $\mathcal{C}(L)$ the set of continuous on $E$ functions of the form $f(x)=\phi\left(\left\langle x, x_{1}^{*}\right\rangle, \ldots,\left\langle x, x_{n}^{*}\right\rangle\right)$, where $x_{j}^{*} \in \mathcal{D}\left(A^{*}\right)$ for all $j=1, \ldots, n$ and $\phi \in C^{2}\left(\mathbb{R}^{n}\right)$ with compact support. It follows from [8] that $\mathcal{C}(L)$ is a core for $L$, and for $f \in \mathcal{C}(L)$,

$$
L f(x)=\frac{1}{2} \operatorname{Tr} D_{H}^{2} f(x)+\langle A x, D f(x)\rangle,
$$

where $D f: E \rightarrow E^{*}$ is the Fréchet derivative of $f$, and $D_{H} f: E \rightarrow H^{*}$ is the Fréchet derivative of $f$ in the direction of $H$ defined by

$$
D_{H} f(x)=\sum_{j=1}^{n} \frac{\partial \phi}{\partial x_{j}}\left(\left\langle x, x_{1}^{*}\right\rangle, \ldots,\left\langle x, x_{n}^{*}\right\rangle\right) B^{*} x_{j}^{*} .
$$

Denote $Q=B B^{*} \in \mathcal{L}\left(E^{*}, E\right)$. When $E$ is a Hilbert space itself, the first term on the right-hand side of (1.4) becomes just $\frac{1}{2} \operatorname{Tr} Q D^{2} f(x)$.

By the result in [9, Thm 5.1], if $E=\mathbb{R}^{n}$ the $L^{1}\left(\mathbb{R}^{n}, \mu_{\infty}\right)$-spectrum of $L$ is equal to $\overline{\mathbb{C}}_{-}=\{\lambda: \operatorname{Re} \lambda \leq 0\}$ with each $\lambda \in \mathbb{C}_{-}=\{\lambda: \operatorname{Re} \lambda<0\}$ being an eigenvalue. This result was extended to infinite-dimensional Banach spaces $E$ in [12] under the assumption of eventual compactness of the semigroup $(S(t))_{t \geq 0}$, and in [2] under the assumption that the part of $A^{*}$ in the reproducing kernel Hilbert space of $\mu_{\infty}$ has an eigenvalue $\gamma \in \mathbb{C}_{-}$. Theorem 1.1 of the present paper generalizes both of these results while requiring less effort to prove it. Also it can serve as an alternative simple coordinate-free proof of the corresponding finite-dimensional result of [9].

Theorem 1.1. If the point spectrum of $A^{*}$ is not empty $\sigma_{p}\left(A^{*}\right) \neq \varnothing$, then the spectrum of the Ornstein-Uhlenbeck operator $L$ coincides with $\overline{\mathbb{C}}_{-}$, and each $\lambda \in \mathbb{C}_{-}$is its eigenvalue.

We remark that due to [11, $\operatorname{Pr} 2.5]$ we have $\sigma_{p}\left(A^{*}\right) \cap\{\lambda: \operatorname{Re} \lambda \geq 0\}=\varnothing$, and thus the condition $\sigma_{p}\left(A^{*}\right) \neq \varnothing$ of Theorem 1.1 is equivalent to the existence of an eigenvalue of $A^{*}$ 
with negative real part. An extension of Theorem 1.1 for the case $\sigma_{p}\left(A^{*}\right)=\varnothing$ seems to be an open question so far.

Corollary 1.2. Under the assumptions of Theorem 1.1, the Ornstein-Uhlenbeck semigroup $(P(t))_{t \geq 0}$ on $L^{1}\left(E, \mu_{\infty}\right)$ is norm discontinuous everywhere.

Proof. Indeed, it easily follows from [7, Thm 4.18] that the spectrum of the generator of an eventually norm continuous semigroup cannot be equal to $\overline{\mathbb{C}}_{-}$.

Acknowledgement. The author would like to thank J.M.A.M. van Neerven and Rostyslav O. Hryniv for helpful remarks.

\section{Proof of Theorem 1.1}

That each $\lambda \in \mathbb{C}_{-}$is an eigenvalue of $L$ we establish in Lemmas 2.1 and 2.3. This implies $\mathbb{C}_{-} \subset \sigma_{p}(L) \subset \sigma(L)$. The fact that $\mathbf{P}$ is contractive on $L^{1}\left(E, \mu_{\infty}\right)$ implies $\sigma(L) \subset \overline{\mathbb{C}}_{-}$. Since the spectrum is closed, this finishes the proof.

By the argument in Section 1, we may assume that $A^{*}$ has an eigenvalue $\gamma \in \mathbb{C}_{-}$. Denote the corresponding eigenvector as $x_{0}^{*} \in E_{\mathbb{C}}^{*}$, where $E_{\mathbb{C}}^{*}$ is the complexification of $E^{*}$.

Lemma 2.1. If $\gamma \in \mathbb{R} \cap \mathbb{C}_{-}$, then each $\lambda \in \mathbb{C}_{-}$is an eigenvalue of $L$.

Proof. Since $\gamma \in \mathbb{R}$, the corresponding eigenvector $x_{0}^{*}$ of $A^{*}$ may be chosen in $E^{*}$. We will show that for each $\lambda \in \mathbb{C}_{-}$we can find an eigenfunction of $L$ of the form $f_{\lambda}(x)=$ $\phi_{\lambda}\left(\left\langle x, x_{0}^{*}\right\rangle\right): E \rightarrow \mathbb{C}$ with some function $\phi_{\lambda}$ on $\mathbb{R}$.

Consider the one-dimensional Ornstein-Uhlenbeck operator defined by

$$
L_{1} \phi(t)=\frac{1}{2} q \phi^{\prime \prime}(t)+\gamma t \phi^{\prime}(t)
$$

for $\phi \in \mathcal{C}\left(L_{1}\right)=\left\{\phi \in C^{2}(\mathbb{R})\right.$ with compact support $\}$, with $q=\left\langle Q x_{0}^{*}, x_{0}^{*}\right\rangle \geq 0$. In fact $\left\langle Q x_{0}^{*}, x_{0}^{*}\right\rangle \neq 0: x_{0}^{*}$ is an eigenvector of $S^{*}$, so (1.2) and $\left\langle Q x_{0}^{*}, x_{0}^{*}\right\rangle=0$ imply $\left\langle Q_{\infty} x_{0}^{*}, x_{0}^{*}\right\rangle=0$ which contradicts the assumption that $\mu_{\infty}$ is nondegenerate. Hence $q>0$.

Here $\mathcal{C}\left(L_{1}\right)$ is viewed as a subspace of $L^{1}\left(\mathbb{R}, \nu_{\infty}\right)$, where $\nu_{\infty}$ is the invariant measure for $L_{1}$. Now observe that $\phi(t) \in L^{1}\left(\mathbb{R}, \nu_{\infty}\right)$ is equivalent to $\phi\left(\left\langle x, x_{0}^{*}\right\rangle\right) \in L^{1}\left(E, \mu_{\infty}\right)$. Indeed, $\nu_{\infty}$ is a centered one-dimensional Gaussian measure with variance $\int_{0}^{\infty} e^{\gamma s} q e^{\gamma s} d s=$ $-\frac{1}{2 \gamma}\left\langle Q x_{0}^{*}, x_{0}^{*}\right\rangle$. By definition, measure $\mu_{\infty}$ on the cylindrical function $\phi\left(\left\langle x, x_{0}^{*}\right\rangle\right)$ is centered one-dimensional Gaussian with variance

$$
\left\langle Q_{\infty} x_{0}^{*}, x_{0}^{*}\right\rangle=\int_{0}^{\infty}\left\langle S(s) Q S^{*}(s) x_{0}^{*}, x_{0}^{*}\right\rangle d s=\int_{0}^{\infty}\left\langle Q e^{\gamma s} x_{0}^{*}, e^{\gamma s} x_{0}^{*}\right\rangle d s=-\frac{1}{2 \gamma}\left\langle Q x_{0}^{*}, x_{0}^{*}\right\rangle .
$$

Hence $\phi(t) \in L^{1}\left(\mathbb{R}, \nu_{\infty}\right)$ if and only if $\phi\left(\left\langle x, x_{0}^{*}\right\rangle\right) \in L^{1}\left(E, \mu_{\infty}\right)$, and $L^{1}\left(\mathbb{R}, \nu_{\infty}\right)$-convergence is equivalent to $L^{1}\left(E, \mu_{\infty}\right)$-convergence for the corresponding $\left\langle x, x_{0}^{*}\right\rangle$-cylindrical functions.

Now, for $\phi \in \mathcal{C}\left(L_{1}\right)$, we have $f(x):=\phi\left(\left\langle x, x_{0}^{*}\right\rangle\right) \in \mathcal{C}(L)$ and

$$
\begin{gathered}
\langle A x, D f(x)\rangle=\left\langle A x, \phi^{\prime}\left(\left\langle x, x_{0}^{*}\right\rangle\right) x_{0}^{*}\right\rangle=\phi^{\prime}\left(\left\langle x, x_{0}^{*}\right\rangle\right)\left\langle x, A^{*} x_{0}^{*}\right\rangle=\gamma \phi^{\prime}\left(\left\langle x, x_{0}^{*}\right\rangle\right)\left\langle x, x_{0}^{*}\right\rangle, \\
\left(D_{H}^{2} f(x)\right)(y)=\phi^{\prime \prime}\left(\left\langle x, x_{0}^{*}\right\rangle\right)\left\langle y, B^{*} x_{0}^{*}\right\rangle B^{*} x_{0}^{*}, \quad y \in H .
\end{gathered}
$$


The only nonzero eigenvalue of the operator on the right-hand side of the last equality is $\phi^{\prime \prime}\left(\left\langle x, x_{0}^{*}\right\rangle\right)\left\langle Q x_{0}^{*}, x_{0}^{*}\right\rangle$, so

$$
L f(x)=\frac{1}{2}\left\langle Q x_{0}^{*}, x_{0}^{*}\right\rangle \phi^{\prime \prime}\left(\left\langle x, x_{0}^{*}\right\rangle\right)+\gamma\left\langle x, x_{0}^{*}\right\rangle \phi^{\prime}\left(\left\langle x, x_{0}^{*}\right\rangle\right)=\left(L_{1} \phi\right)\left(\left\langle x, x_{0}^{*}\right\rangle\right) .
$$

For each $\lambda \in \mathbb{C}_{-}$let $\phi_{\lambda}(t) \in L^{1}\left(\mathbb{R}, \nu_{\infty}\right)$ be an eigenfunction of $L_{1}$ corresponding to the eigenvalue $\lambda$ (we use the one-dimensional case of [9, Thm 5.1]). Now we find $\phi_{n}(t) \in \mathcal{C}\left(L_{1}\right)$ with $\phi_{n} \rightarrow \phi_{\lambda}$ in $L^{1}\left(\mathbb{R}, \nu_{\infty}\right)$. Then $\phi_{n}\left(\left\langle x, x_{0}^{*}\right\rangle\right) \in \mathcal{C}(L)$ and $\phi_{n}\left(\left\langle x, x_{0}^{*}\right\rangle\right) \rightarrow \phi_{\lambda}\left(\left\langle x, x_{0}^{*}\right\rangle\right)$ in $L^{1}\left(E, \mu_{\infty}\right)$, and applying (2.1) to $\phi_{n}\left(\left\langle x, x_{0}^{*}\right\rangle\right)$ 's we obtain $f_{\lambda}(x):=\phi_{\lambda}\left(\left\langle x, x_{0}^{*}\right\rangle\right) \in \mathcal{D}(L)$ with $L f_{\lambda}(x)=\lambda f_{\lambda}(x)$.

We state an easy auxiliary lemma, the proof of which is left to the reader.

Lemma 2.2. Let $H$ be a Hilbert space, $x_{1}, x_{2}, y_{1}, y_{2} \in H$. The trace of the operator $A x=\left\langle x, y_{1}\right\rangle x_{1}+\left\langle x, y_{2}\right\rangle x_{2}$ is equal to $\operatorname{Tr} A=\left\langle x_{1}, y_{1}\right\rangle+\left\langle x_{2}, y_{2}\right\rangle$.

Now we prove

Lemma 2.3. Let $\gamma \in \mathbb{C}_{-} \backslash \mathbb{R}$. Then each $\lambda \in \mathbb{C}_{-}$is an eigenvalue of $L$.

Proof. Let $\gamma=a+b i, a<0, b \neq 0$. Take $h_{1}^{*}:=\operatorname{Re} x_{0}^{*} \in E^{*}, h_{2}^{*}:=\operatorname{Im} x_{0}^{*} \in E^{*}$. We have $A^{*} h_{1}^{*}=a h_{1}^{*}-b h_{2}^{*}, A^{*} h_{2}=b h_{1}^{*}+a h_{2}^{*}$, and also

$$
\begin{aligned}
S^{*}(s) h_{1}^{*} & =e^{a s}\left(h_{1}^{*} \cos b s-h_{2}^{*} \sin b s\right), \\
S^{*}(s) h_{2}^{*} & =e^{a s}\left(h_{1}^{*} \sin b s+h_{2}^{*} \cos b s\right) .
\end{aligned}
$$

We follow the same approach as in Lemma 2.1. Consider the two-dimensional OrnsteinUhlenbeck operator

$$
L_{2} \phi(t):=\frac{1}{2} \operatorname{Tr}\left(R D^{2} \phi(t)\right)+\langle C t, D \phi(t)\rangle, \quad t \in \mathbb{R}^{2}
$$

for $\phi \in \mathcal{C}\left(L_{2}\right)=\left\{\phi \in C^{2}\left(\mathbb{R}^{2}\right)\right.$ with compact support $\}$, where

$$
R:=\left(r_{i j}\right)_{i, j=1}^{2}=\left(\left\langle Q h_{i}^{*}, h_{j}^{*}\right\rangle\right)_{i, j=1}^{2}, \quad C:=\left(c_{i j}\right)_{i, j=1}^{2}=\left(\begin{array}{cc}
a & -b \\
b & a
\end{array}\right) .
$$

Now we show that the covariance operator $R_{\infty}$ of the invariant measure $\nu_{\infty}$ corresponding to $L_{2}$ is the same as of the image measure of $\mu_{\infty}$ under the map $\left(\left\langle x, h_{1}^{*}\right\rangle,\left\langle x, h_{2}^{*}\right\rangle\right)$. This can be verified directly: using (2.2) and (2.3), it is easy to show that the matrix $e^{C s} R e^{C^{*} s}$ equals to the matrix $\left(\left\langle S(s) Q S^{*}(s) h_{i}^{*}, h_{j}^{*}\right\rangle\right)_{i, j=1}^{2}$, and thus

$$
\begin{aligned}
R_{\infty}=\int_{0}^{\infty} e^{C s} R e^{C^{*} s} d s & =\left(\int_{0}^{\infty}\left\langle S(s) Q S^{*}(s) h_{i}^{*}, h_{j}^{*}\right\rangle d s\right)_{i, j=1}^{2} \\
& =\left(\left\langle Q_{\infty} h_{i}^{*}, h_{j}^{*}\right\rangle\right)_{i, j=1}^{2} .
\end{aligned}
$$

This implies that $\phi(t) \in L^{1}\left(\mathbb{R}^{2}, \nu_{\infty}\right)$ if and only if $\phi\left(\left\langle x, h_{1}^{*}\right\rangle,\left\langle x, h_{2}^{*}\right\rangle\right) \in L^{1}\left(E, \mu_{\infty}\right)$, and $L^{1}\left(\mathbb{R}^{2}, \nu_{\infty}\right)$-convergence is equivalent to $L^{1}\left(E, \mu_{\infty}\right)$-convergence for the corresponding $\left(\left\langle x, h_{1}^{*}\right\rangle,\left\langle x, h_{2}^{*}\right\rangle\right)$-cylindrical functions. 
Now, for $\phi \in \mathcal{C}\left(L_{2}\right)$, we have $f(x):=\phi\left(\left\langle x, h_{1}^{*}\right\rangle,\left\langle x, h_{2}^{*}\right\rangle\right) \in \mathcal{C}(L)$ and

$$
\begin{aligned}
& \langle A x, D f(x)\rangle=\left\langle A x, \frac{\partial \phi}{\partial t_{1}}\left(\left\langle x, h_{1}^{*}\right\rangle,\left\langle x, h_{2}^{*}\right\rangle\right) h_{1}^{*}\right\rangle+\left\langle A x, \frac{\partial \phi}{\partial t_{2}}\left(\left\langle x, h_{1}^{*}\right\rangle,\left\langle x, h_{2}^{*}\right\rangle\right) h_{2}^{*}\right\rangle \\
& =\frac{\partial \phi}{\partial t_{1}}\left(\left\langle x, h_{1}^{*}\right\rangle,\left\langle x, h_{2}^{*}\right\rangle\right)\left(a\left\langle x, h_{1}^{*}\right\rangle-b\left\langle x, h_{2}^{*}\right\rangle\right)+\frac{\partial \phi}{\partial t_{2}}\left(\left\langle x, h_{1}^{*}\right\rangle,\left\langle x, h_{2}^{*}\right\rangle\right)\left(b\left\langle x, h_{1}^{*}\right\rangle+a\left\langle x, h_{2}^{*}\right\rangle\right),
\end{aligned}
$$

and, for $y \in H$,

$$
\begin{gathered}
\left(D_{H}^{2} f(x)\right)(y)=\frac{\partial^{2} \phi}{\partial t_{1}^{2}}\left(\left\langle x, h_{1}^{*}\right\rangle,\left\langle x, h_{2}^{*}\right\rangle\right)\left\langle y, B^{*} h_{1}^{*}\right\rangle B^{*} h_{1}^{*}+\frac{\partial^{2} \phi}{\partial t_{1} \partial t_{2}}\left(\left\langle x, h_{1}^{*}\right\rangle,\left\langle x, h_{2}^{*}\right\rangle\right)\left\langle y, B^{*} h_{2}^{*}\right\rangle B^{*} h_{1}^{*} \\
+\frac{\partial^{2} \phi}{\partial t_{2} \partial t_{1}}\left(\left\langle x, h_{1}^{*}\right\rangle,\left\langle x, h_{2}^{*}\right\rangle\right)\left\langle y, B^{*} h_{1}^{*}\right\rangle B^{*} h_{2}^{*}+\frac{\partial^{2} \phi}{\partial t_{2}^{2}}\left(\left\langle x, h_{1}^{*}\right\rangle,\left\langle x, h_{2}^{*}\right\rangle\right)\left\langle y, B^{*} h_{2}^{*}\right\rangle B^{*} h_{2}^{*} .
\end{gathered}
$$

Taking into account Lemma 2.2 , we get

$$
\begin{array}{r}
L f(x)=\frac{1}{2} \sum_{i, j=1}^{2} r_{i j} \frac{\partial^{2} \phi}{\partial t_{i} \partial t_{j}}\left(\left\langle x, h_{1}^{*}\right\rangle,\left\langle x, h_{2}^{*}\right\rangle\right)+\sum_{i, j=1}^{2} c_{i j}\left\langle x, h_{j}^{*}\right\rangle \frac{\partial \phi}{\partial t_{i}}\left(\left\langle x, h_{1}^{*}\right\rangle,\left\langle x, h_{2}^{*}\right\rangle\right) \\
\left.=\left(L_{2} \phi\right)\left(\left\langle x, h_{1}^{*}\right\rangle,\left\langle x, h_{2}^{*}\right\rangle\right)\right) .
\end{array}
$$

Now observe that $\sigma(C)=\{a \pm i b\} \subset \mathbb{C}_{-}$, and the kernel of $R$ does not contain any invariant subspace of $C^{*}$ : by (2.4) this would imply degeneracy of $R_{\infty}$ and $Q_{\infty}$, and consequently of $\mu_{\infty}$. Thus we can use [9, Thm 5.1] to conclude that for any $\lambda \in \mathbb{C}_{-}$there exists an eigenfunction $\phi_{\lambda}(t) \in L^{1}\left(\mathbb{R}^{2}, \nu_{\infty}\right)$ of $L_{2}$ corresponding to $\lambda$. Approximating $\phi_{\lambda}$ by $\phi_{n} \in \mathcal{C}\left(L_{2}\right)$, $\phi_{n} \rightarrow \phi_{\lambda}$ in $L^{1}\left(\mathbb{R}^{2}, \nu_{\infty}\right)$, and then applying (2.5) to $f_{n}(x):=\phi_{n}\left(\left\langle x, h_{1}^{*}\right\rangle,\left\langle x, h_{2}^{*}\right\rangle\right) \in \mathcal{C}(L)$, we obtain $f_{n}(x) \rightarrow \phi_{\lambda}\left(\left\langle x, h_{1}^{*}\right\rangle,\left\langle x, h_{2}^{*}\right\rangle\right)=: f_{\lambda}(x)$ in $L^{1}\left(E, \mu_{\infty}\right), L f_{n}(x) \rightarrow \lambda f_{\lambda}(x)$ in $L^{1}\left(E, \mu_{\infty}\right)$. Hence $f_{\lambda}(x) \in \mathcal{D}(L)$ and $L f_{\lambda}(x)=\lambda f_{\lambda}(x)$.

\section{REFERENCES}

[1] Z. Brzeźniak, J.M.A.M. van Neerven, Stochastic convolution in separable Banach spaces and the stochastic linear Cauchy problem, Studia Math. 143 (2000), pp. 43-74.

[2] A. Chojnowska-Michalik, On $L^{1}(H, \mu)$-properties of Ornstein-Uhlenbeck semigroups, Stochastic partial differential equations and applications-VII, Lect. Notes Pure Appl. Math., 245, Chapman \& Hall/CRC, Boca Raton, FL, 2006, pp. 77-88.

[3] A. Chojnowska-Michalik, B. Goldys, Existence, uniqueness and invariant measures for stochastic semilinear equations on Hilbert spaces, Probab. Theory Relat. Fields 102 (1995), pp. 331-356.

[4] A. Chojnowska-Michalik, B. Goldys, On regularity properties of nonsymmetric OrnsteinUhlenbeck semigroups in $L^{p}$ spaces, Stoch. Stoch. Rep. 59 (1996), pp. 183-209.

[5] G. Da Prato, J. ZabczyK, "Second order partial differential equations in Hilbert spaces", Cambridge University Press, Cambridge, 2002.

[6] G. Da Prato, J. Zabczyk, "Stochastic equations in infinite dimensions", Cambridge University Press, Cambridge, 1992.

[7] K.J. Engel, R. Nagel, "One-Parameter Semigroups for Linear Evolution Equations", SpringerVerlag, New York, 2000.

[8] B. Goldys, J.M.A.M. NeERven, Transition semigroups of Banach space valued OrnsteinUhlenbeck processes, Acta Appl. Math. 76 (2003), pp. 283-330.

[9] G. Metafune, D. Pallara, E. Priola, Spectrum of Ornstein-Uhlenbeck operators in $L^{p}$ spaces with respect to invariant measures, J. Funct. Anal. 196 (2002), pp. 40-60. 
[10] J.M.A.M. VAN NEERVEn, Second quantization and the $L^{p}$-spectrum of nonsymmetric OrnsteinUhlenbeck operators, Infin. Dimens. Anal. Quantum Probab. Relat. Top. 8 (2005), pp. 473-496.

[11] J.M.A.M. VAN NEERVEN, Uniqueness of invariant measures for the stochastic Cauchy problem in Banach spaces, Operator theory: Advances and Applications, 127 (2001), pp. 491-517.

[12] J.M.A.M. van Neerven, E. Priola, Norm discontinuity and spectral properties of OrnsteinUhlenbeck semigroups, J. Evolution Equations 5 (2005), pp. 557-576.

[13] K. YosidA, "Functional Analysis", Springer-Verlag, Berlin-Göttingen-Heidelberg, 1965.

Dept of Mathematics, California Institute of Technology, MC 253-37, 1200 E.California Blvd, Pasadena, CA 91125, US.

TElephone: (626)29-89-119

E-MAIL: ROSTYSLA@CALTECH.EDU 